\title{
Splat Shapes in a Thermal Spray Coating Process: Simulations and Experiments
}

\author{
M. Pasandideh-Fard, V. Pershin, S. Chandra, and J. Mostaghimi
}

(Submitted 24 October 2000; in revised form 16 February 2001)

\begin{abstract}
We studied the deposition of nickel particles in a plasma spray on a stainless steel surface using both experiments and numerical simulations. We developed a three-dimensional computational model of free-surface fluid flow that includes heat transfer and solidification and used it to simulate the impact of nickel particles. In our experiments, particles landing on a polished stainless steel surface at a temperature below $300{ }^{\circ} \mathrm{C}$ splashed and formed irregular splats, whereas those deposited on substrates heated above $400{ }^{\circ} \mathrm{C}$ formed round disk splats. Simulations showed that formation of fingers around the periphery of a spreading drop is caused by the presence of a solid layer. Droplets that spread completely before the onset of solidification will not splash. To sufficiently delay the instant at which solidification started in our simulations to obtain disk splats, we had to increase the thermal contact resistance between the droplet and the substrate by an order of magnitude. We measured the thickness of the oxide layer on the test surfaces used in our experiments and confirmed that heating them creates an oxide layer on the surface that increases the thermal contact resistance. We demonstrated that the numerical model could be used to simulate the deposition of multiple droplets on a surface to build up a coating.
\end{abstract}

Keywords 3D numerical model, droplet impact, free surface flows, solidification, thermal spray coating

\section{Introduction}

Thermal spray coating is a term used to describe a family of processes that employs a heat source (either electrical or combustion based) to melt powders of metallic and nonmetallic materials and spray them with high velocity onto a substrate, forming a dense deposit. This technology is commonly used to apply protective coatings on components to shield them from wear, corrosion, and thermal shock. Operation of industrial thermal spray coating equipment requires considerable skill because many of the equipment settings are user selectable, such as the power supplied to the gun, the powder feed rate, and the distance between the gun and the substrate. To obtain good coatings these settings have to be chosen carefully, and because they could differ for each substrate-coating combination, much trial and error goes into optimizing them. In recent years a great deal of research has been devoted to trying to understand exactly how variations made to spray parameters changes coating properties.

Thermal spray coatings are built up by the accumulation of splats formed by the deposition of individual molten droplets. Coating properties such as porosity, adhesion strength, and roughness depend on the shape of these splats and how they bond with each other and with the substrate. The form that splats assume after freezing is a function of process parameters such as in-flight particle size distribution, velocity, temperature, and

M. Pasandideh-Fard, V. Pershin, S. Chandra, and J. Mostaghimi, University of Toronto, Department of Mechanical and Industrial Engineering, 5 King's College Rd., Toronto, Canada M5S 3G8. Contact email: mostag@mie.utoronto.ca. degree of solidification, and substrate material and temperature. ${ }^{[1]}$

The temperature of the substrate, on which molten droplets land, has been found to have an important effect on splat shape. Bianchi et al. ${ }^{[2]}$ took scanning electron micrographs of individual alumina and zirconia splats deposited by plasma torches on a stainless steel plate. They found that droplets landing on a cold substrate (with a temperature below $100^{\circ} \mathrm{C}$ ) splashed extensively after impact so that the splats were irregular in shape with fingers of material radiating out from their center. Splats deposited on a hot surface (above $150^{\circ} \mathrm{C}$ ) were almost perfectly circular, and were shaped like disks. Fukomoto et al. ${ }^{[3]}$ sprayed nickel powders on substrates of different materials, and confirmed that there was a "transition temperature" above which disk splats were obtained. The transition temperature depended on surface material properties and increased with substrate thermal conductivity. They speculated that splashing is caused by freezing of the molten droplet as it spread on the substrate following impact: the formation of a solid layer at the dropletsubstrate interface triggers radial jetting of the liquid and makes it splash. Recent experiments have shown that the transition temperature is not a material property alone, but depends on a number of parameters, including the particle-substrate material combination, ${ }^{[4]}$ surface contamination, ${ }^{[5]}$ and surface oxidation. ${ }^{[6]}$

Splat morphology has been shown to have an important effect on coating qualities. Sakakibara et al. ${ }^{[7]}$ plasma sprayed yttria-stabilized zirconia particles on a polished stainless steel surface and confirmed that the transition to disk splats occurred at a substrate temperature of $150-200^{\circ} \mathrm{C}$. Coatings deposited on surfaces above this transition temperature, so that they consisted mostly of disks splats, showed a significant increase in adhesion strength.

Despite all of the research done into splat formation, there is no consensus on the mechanism that leads to the transition from 
irregular-shaped splats to disk splats, or what combination of impact parameters determines the transition temperature. A better understanding of the process that causes droplet splashing will allow us to determine the optimum temperature at which substrates should be maintained during spraying to give the best coating quality. Therefore, the objectives of our study were to (1) perform controlled experiments in which particles in a plasma spray were deposited on a surface; (2) simulate droplet impact using a numerical model; and (3) determine conditions under which droplets splashed during impact in both experiments and simulations.

We sprayed nickel powders with a dc plasma torch (SG-100, Miller Thermal, Appleton, WI) onto a stainless steel substrate, the temperature of which could be accurately controlled. We also used diagnostic instruments (DPV-2000, Tecnar Automation, Ltd., Montreal, Canada) to measure the velocity, size, and temperature of particles prior to impact, and recorded splat shapes using an electron microscope.

Simulating the impact and splashing of a molten droplet requires the development of a fully three-dimensional (3D) freesurface flow model that includes heat transfer and solidification. Several numerical models of droplet impact on a surface have been developed. However, most of these works ${ }^{[8-12]}$ have focused on the axisymmetric, or two-dimensional impact of a droplet on a surface. But a splashing droplet, which has fingers of liquid radiating from it, is not axisymmetric and must be modeled in all three spatial dimensions. Bussmann et al. ${ }^{[13]}$ developed a 3D numerical model of free-surface flows and demonstrated that it could be used to model splashing of liquid droplets.

\begin{tabular}{|ll|}
\hline \multicolumn{1}{|c|}{ Nomenclature } \\
\hline$C$ & specific heat \\
$f$ & volume of fluid fraction \\
$\vec{F}_{\mathrm{b}}$ & body force \\
$h$ & enthalpy \\
$H_{\mathrm{f}}$ & latent heat of fusion \\
$k$ & thermal conductivity \\
$p$ & pressure \\
$q$ & heat flux \\
$R_{\mathrm{c}}$ & thermal contact resistance at droplet/substract interface \\
$t$ & time \\
$T$ & temperature \\
$T_{\mathrm{m}}$ & droplet melting point \\
$\vec{V}$ & velocity \\
& \\
\hline$\beta$ & $k / C$ \\
$\Theta$ & liquid volume fraction \\
$v$ & kinematic viscosity \\
$\rho$ & density \\
$\phi$ & source term in energy equation $\left(H_{\mathrm{f}} k_{\mathrm{l}} / C_{\mathrm{l}}\right)$ \\
& \\
\hline 1 & liquid \\
$\mathrm{s}$ & solid \\
$\mathrm{w}$ & substrate \\
\hline
\end{tabular}

Their model did not consider any heat transfer and all of the cases they examined were of isothermal impact. In this study, we have extended the fluid flow model of Bussmann et al. ${ }^{[13]}$ to include heat transfer and solidification. We used the model to predict the shapes of splats formed during the impact of molten nickel particles on a stainless steel substrate under various impact conditions.

\section{Numerical Method}

\subsection{Fluid Flow}

Fluid flow in an impacting droplet is modeled using a finite difference solution of the Navier-Stokes equations in a 3D Cartesian coordinate system. The ambient air during droplet impact is assumed to be dynamically inactive, i.e., only the liquid phase is considered. Other assumptions are that the liquid is incompressible with a constant value of surface tension, and the fluid flow is laminar. The flow Reynolds number (assuming radial flow over a flat plate in the droplet after impact) was estimated to be at most $10^{4}$, which is too small to induce turbulence. The surface profile of the deforming droplet is defined using the "fractional volume of fluid" scheme. In this method, a scalar function $f$ is defined as the fraction of a cell volume occupied by fluid. $f$ is assumed to be unity when a cell is fully occupied by the fluid and zero for an empty cell. Cells with values of $0<f<1$ contain a free surface. The volume-tracking algorithm used for advection of function $f$ consists of two steps: an approximate reconstruction of the interface followed by a geometric evaluation of volume fluxes across cell faces. The free surface is reconstructed by locating a plane within each free surface cell such that the exact value of $f$ is conserved and the unit normal to this plane is directed into the liquid. Surface tension is modeled as a volume force acting on fluid near the free surface; the method used is the continuum surface force (CSF) model integrated with smoothed values of function $f$ in evaluating free surface curvature. Tangential stresses at the free surface are neglected. Contact angles are applied as a boundary condition at the contact line.

The above methods for advection of function $f$ and application of surface tension on the free surface are only for rectangular mesh that necessitates employing a Cartesian coordinate system. When a Cartesian mesh is used to simulate a radial flow, a slight squaring effect results for a coarse grid; the reason for the squaring is related to the difficulty in specifying velocities as a boundary condition at a curved interface. The squaring effect diminishes as the computational mesh becomes finer. Details of the fluid flow model are given by Bussmann et al. ${ }^{[13]}$ Liquid density and surface tension were assumed to be constant. Liquid viscosity and substrate thermal properties, however, were assumed to vary with temperature.

\subsection{Heat Transfer}

Heat transfer in the droplet was modeled by solving the energy equation, neglecting viscous dissipation; densities of liquid and solid were assumed to be constant and equal to each other:

$\frac{\partial h}{\partial t}+(\vec{V} \cdot \vec{\nabla}) h=\frac{1}{\rho} \vec{\nabla} \cdot(k \vec{\nabla} T)$ 


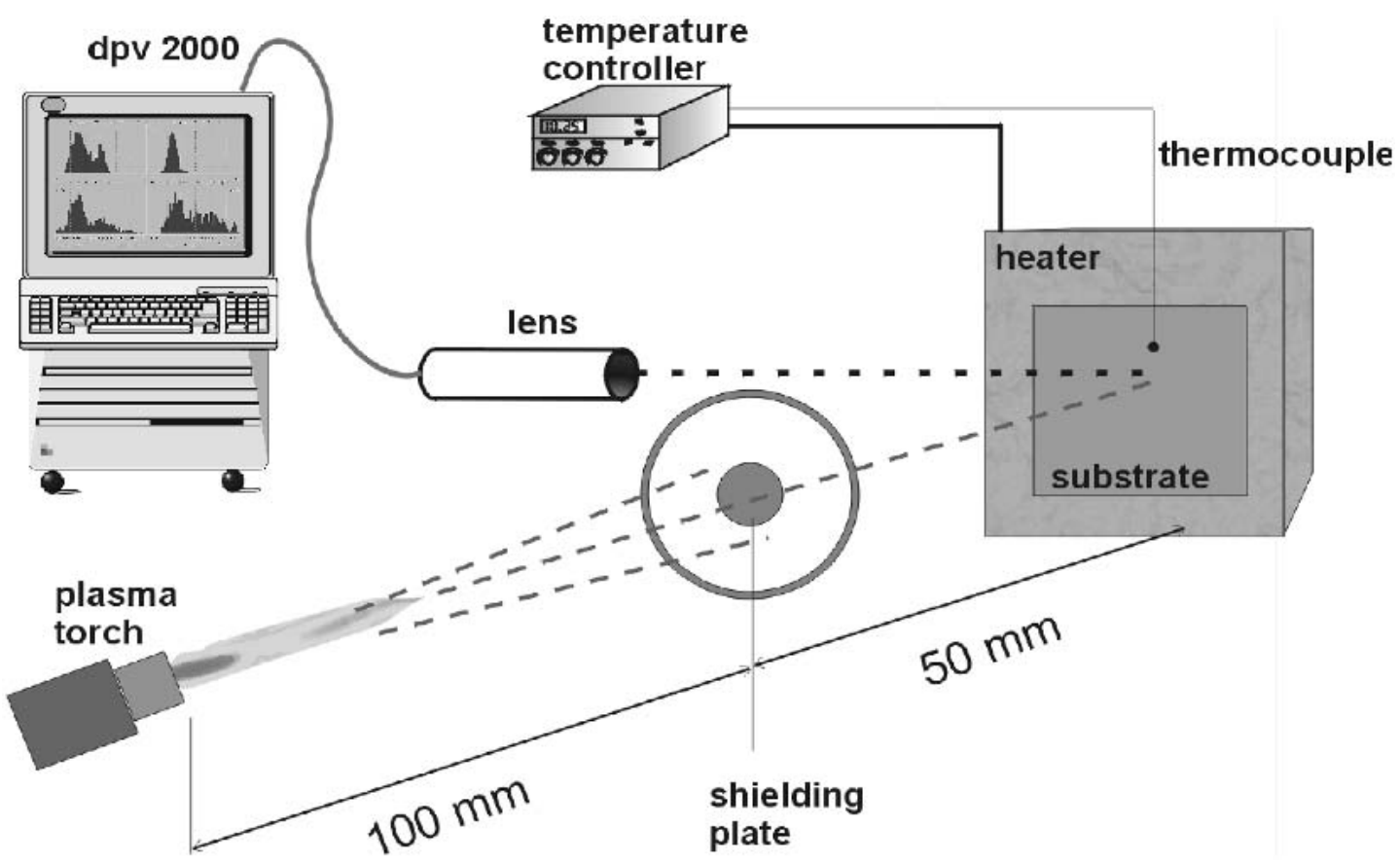

Fig. 1 Schematic of the experimental apparatus

where $\vec{V}$ represents the velocity vector, $T$ is the temperature, $h$ is the enthalpy, $\rho$ is the density, and $k$ is the thermal conductivity of the droplet. Because the energy equation has two dependent variables - temperature $T$ and enthalpy $h$-we used the enthalpy transforming mode ${ }^{[14]}$ to convert the energy equation to an equation with only one dependent variable: the enthalpy. The main advantage of this method is that it solves energy equation for both phases simultaneously. The final form, given by Pasandideh-Fard, ${ }^{[15]}$ is

$\frac{\partial h}{\partial t}+(\vec{V} \cdot \vec{\nabla}) h=\frac{1}{\rho} \nabla^{2}(\beta h)+\frac{1}{\rho} \nabla^{2} \phi$

where in the solid phase,

$h \leq 0 ; \quad \beta=\frac{k_{\mathrm{s}}}{C_{\mathrm{s}}}, \phi=0$

at the liquid-solid interface,

$0<h<H_{\mathrm{f}} ; \quad \beta=0, \phi=0$

and in the liquid phase,

$h \geq H_{\mathrm{f}} ; \quad \beta=\frac{k_{\mathrm{l}}}{C_{1}}, \phi=-\frac{H_{\mathrm{f}} k_{\mathrm{l}}}{C_{1}}$

where $C$ is the specific heat, $H_{\mathrm{f}}$ is the latent heat of fusion, and $\phi$ is a new source term. The energy equation now has only one dependent variable, the enthalpy $h$. The relationship between temperature and enthalpy is given by

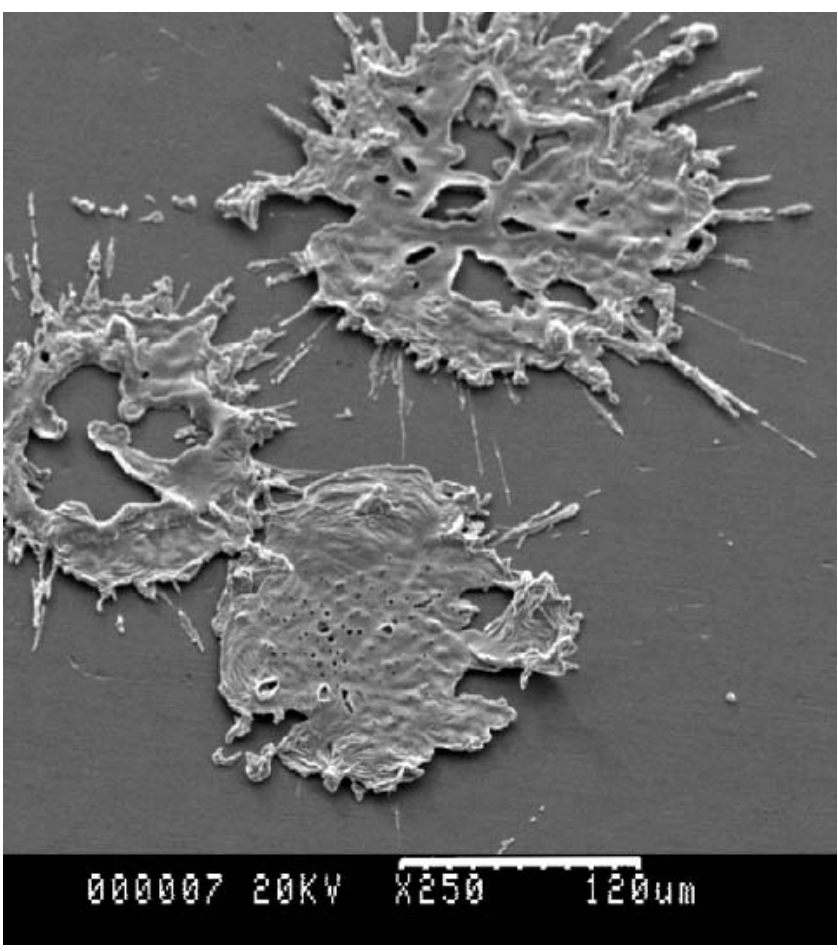

Fig. 2 Nickel splat deposited on a stainless steel surface initially at 290 ${ }^{\circ} \mathrm{C}$. The splats are irregular, showing evidence of splashing as they spread.

$T=T_{\mathrm{m}}+\frac{1}{k}(\beta h+\phi)$

where $T_{\mathrm{m}}$ is the melting point of the droplet. Heat transfer within the substrate is by conduction only; the governing equation is 
$\rho_{\mathrm{w}} C_{\mathrm{w}} \frac{\partial T_{\mathrm{w}}}{\partial t}=\vec{\nabla} \cdot\left(k_{\mathrm{w}} \vec{\nabla} T_{\mathrm{w}}\right)$

At the free surface of the droplet, we imposed an adiabatic boundary condition. This condition must be supplemented with the specification of enthalpy $h$ and functions $\beta$ and $\phi$ immediately outside the surface, where these values are needed in the finite-difference approximations for points located at the free surface. This condition can easily be modified to a convective or radiative boundary condition. For the cases under consideration, the dominant heat transfer was due to conduction to the substrate at the initial stages of impact, and conduction and convection within the droplet at the later stages of impact. Estimates of heat loss by convection from the droplet surface to the surrounding gas showed them to be three orders of magnitude lower than that to the substrate. Therefore, the adiabatic condition at the free surface was reasonable. Thermal contact resistance between the droplet and substrate $\left(R_{\mathrm{c}}\right)$ is defined by

$R_{\mathrm{c}}=\frac{\left(T-T_{\mathrm{w}}\right)_{\text {substrate }}}{q}$

where $q$ is the heat flux from the droplet to substrate. Values of $R_{\mathrm{c}}$ were provided as an input to the model. Although in principle, $R_{\mathrm{c}}$ could vary with both time and position on the interface, we used a constant value in the simulations.

\subsection{Solidification}

In the presence of a solid phase, computations of the velocity field have to account for the presence of a moving, irregularly

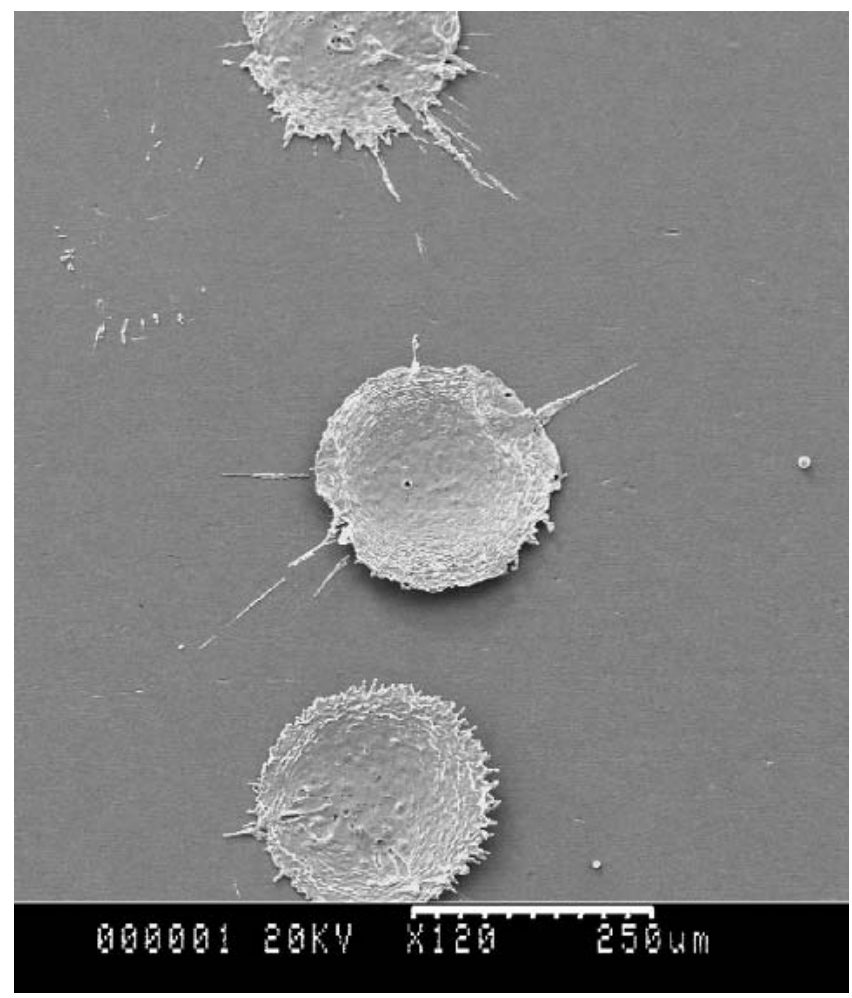

Fig. 3 Nickel splat deposited on a stainless steel surface initially at $340{ }^{\circ} \mathrm{C}$. The splats are less irregular than they were on a surface at $290^{\circ} \mathrm{C}$. shaped solidification front on which the relevant boundary conditions have to be applied. We treat the solidified regions of the domain using a modified version of the fixed velocity method. In this approach, a liquid volume fraction $\Theta$ is defined such that $\Theta$ $=1$ for a cell containing only liquid, $\Theta=0$ for a cell containing only solid, and $0<\Theta<1$ for a cell containing a portion of the solidification front. Normal and tangential velocities on the faces of cells containing only solidified material are set to zero. On the basis of this method, the final modified continuity and momentum equations are ${ }^{[15]}$

$\vec{\nabla} \cdot(\Theta \vec{V})=0$

$\frac{\partial(\Theta \vec{V})}{\partial t}+(\Theta \vec{V} \cdot \vec{\nabla}) \vec{V}=\frac{-\Theta}{\rho} \vec{\nabla} p+\Theta v \nabla^{2} \vec{V}+\frac{\Theta}{\rho} \overrightarrow{F_{\mathrm{b}}}$

$\frac{\partial f}{\partial t}+(\Theta \vec{V} \cdot \vec{\nabla}) f=0$

where $p$ is the pressure, $v$ is the kinematic viscosity, and $F_{\mathrm{b}}$ is any body forces acting on the fluid.

\subsection{Numerical Procedure}

The modified Navier-Stokes equations were solved on a Eulerian, rectangular, staggered mesh in a $3 \mathrm{D}$ Cartesian coordinate

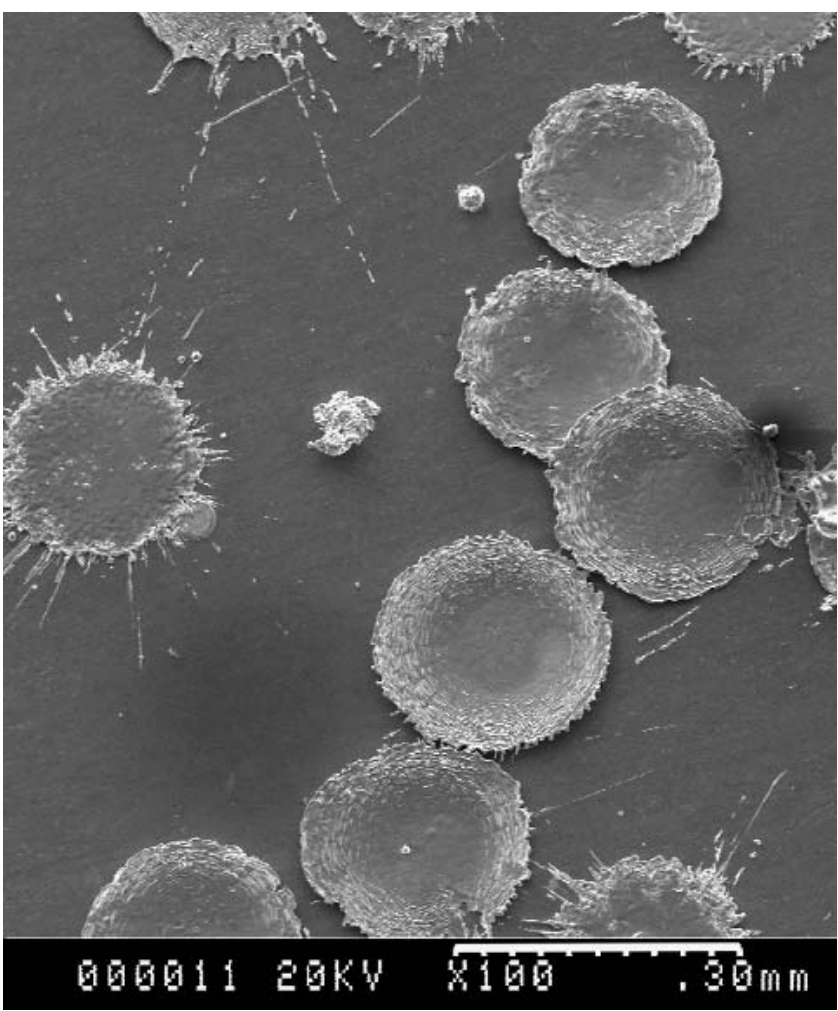

Fig. 4 Nickel splat deposited on a stainless steel surface initially at 400 ${ }^{\circ} \mathrm{C}$. Most of the splats are disk shaped, with little sign of splashing during impact. 

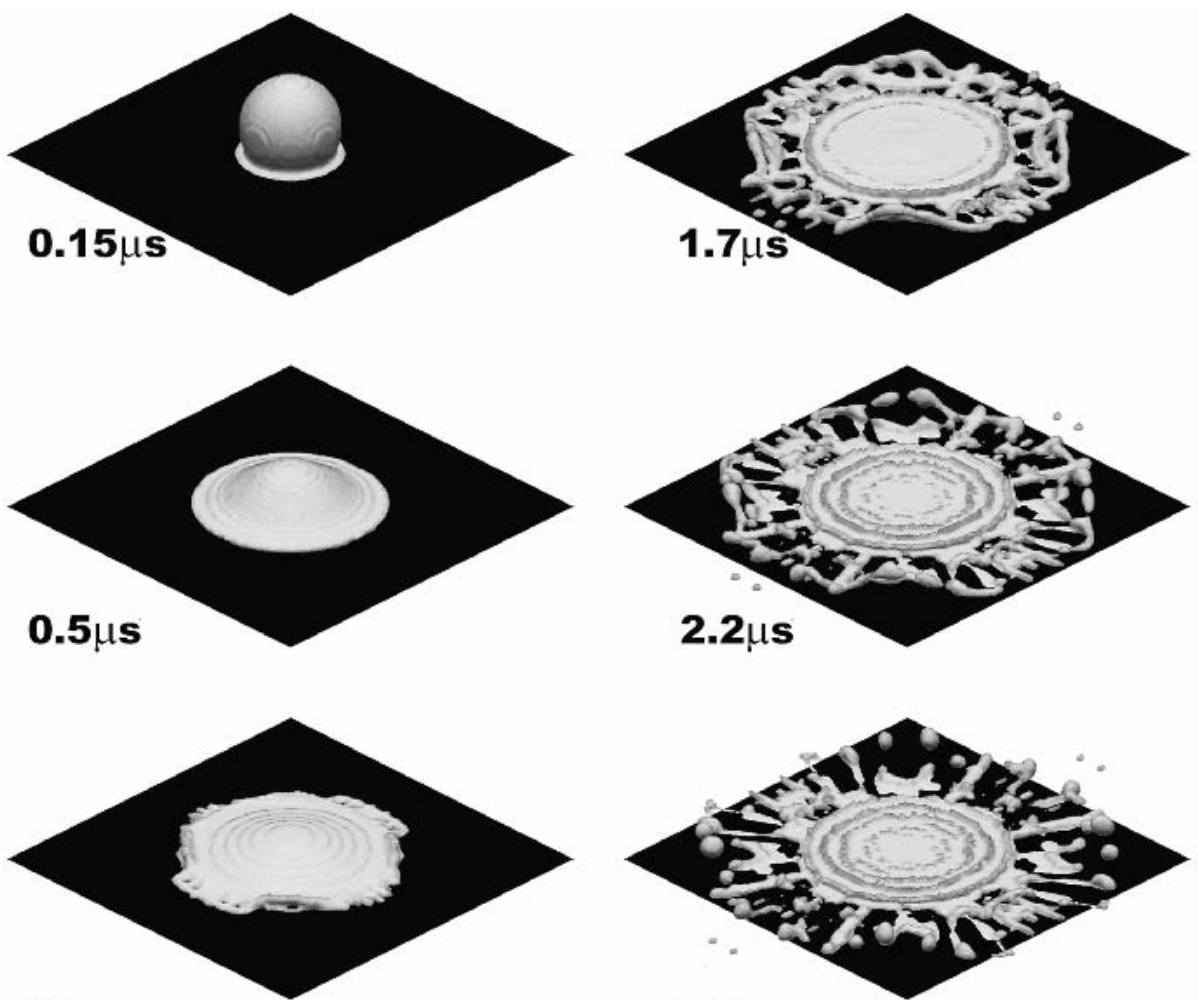

$0.8 \mu \mathrm{s}$
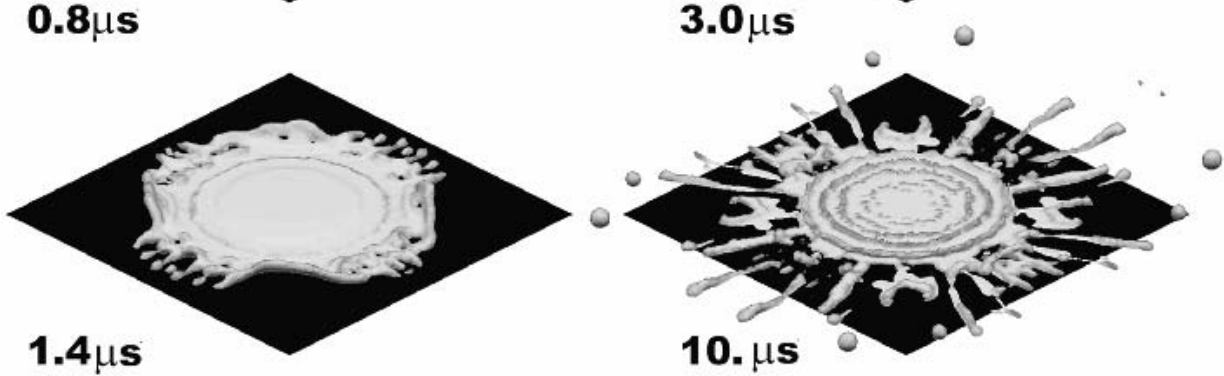

Fig. 5 Simulations showing the impact of a $60 \mu \mathrm{m}$ diameter molten nickel particle at $1600{ }^{\circ} \mathrm{C}$ landing with a velocity of $73 \mathrm{~m} / \mathrm{s}$ on a stainless steel plate initially at a temperature of $290^{\circ} \mathrm{C}$. The contact resistance at the substrate surface was assumed to be $10^{-7} \mathrm{~m}^{2} \mathrm{~K} / \mathrm{W}$.

system. The computational procedure for advancing the solution through one time step is as follows:

1) From time level $n$ values, the velocity and pressure fields as well as $f$ are calculated at time level $n+1$ in accordance with the 3D model of Bussmann et al. ${ }^{[13]}$

2) Given the droplet enthalpy and substrate temperature fields at time level $n, \mathrm{Eq} 2$ and 5 are solved implicitly to obtain the new enthalpy field in the droplet and the new temperature field in the substrate. Temperatures in the droplet can then be calculated from $\mathrm{Eq} 4$.

3) New values of the liquid volume fraction $\Theta$ are calculated from the enthalpy field in the droplet by using Eq 3(a-c) in conjunction with an algorithm described by Voller and Cross. ${ }^{[16]}$ In this algorithm, while a change of phase is occurring in the subregion of a computational cell, the rate of change in the cell enthalpy equals the velocity of the phase change front across the subregion multiplied by the latent heat of fusion.

4) Flow and thermal boundary conditions are imposed on the free surface, at the solidification front, and at the bound- aries of the computational domain. In particular, the thermal contact resistance at the droplet-substrate interface is applied using Eq 6 to calculate the heat flux from the droplet. This value of $q$ is then used to update temperature boundary conditions along the bottom surface of the droplet and the upper plane of the substrate.

Repetition of these steps allowed advancing the solution through an arbitrary time interval. The droplet was discretized with a grid spacing equal to $1 / 15$ of the droplet radius. The substrate mesh had the same resolution and was extended far enough that its boundaries could be assumed at constant temperature. Numerical computations were performed on a Sun U1tra Enterprise 450 workstation (Sun Microsystems, Inc., Palo Alto, CA). A typical central processing unit (CPU) time was $40 \mathrm{~h}$.

\section{Experimental Method}

To validate our numerical model, we carried out experiments in which nickel powder was plasma-sprayed onto a stainless 

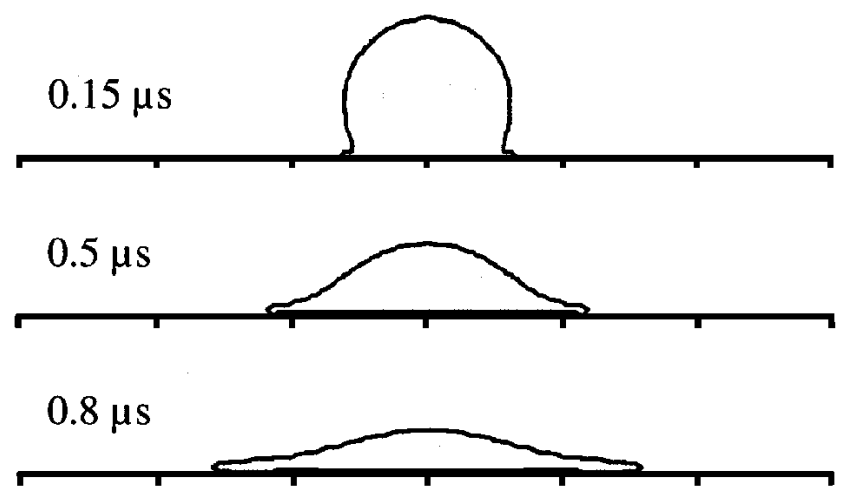

\section{$1.1 \mu \mathrm{s}$}

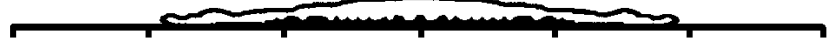

$1.4 \mu \mathrm{s}$

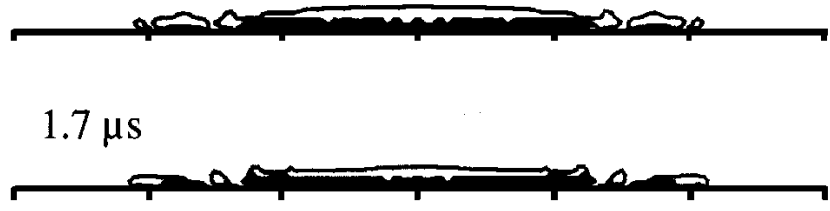

$2.2 \mu \mathrm{s}$

10. $\mu \mathrm{s}$

Fig. 6 A cross-sectional view of the images in Fig. 5. Black shows the solidified portion of the droplet and white represents liquid.

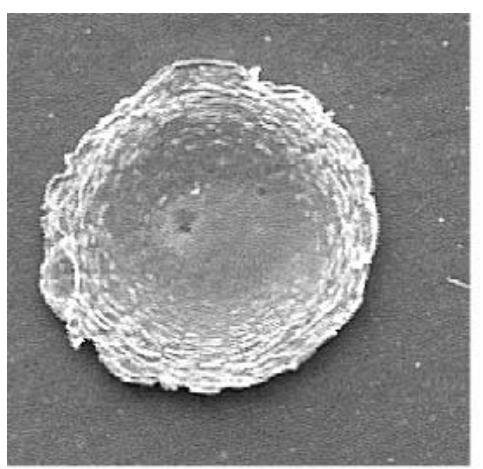

a)
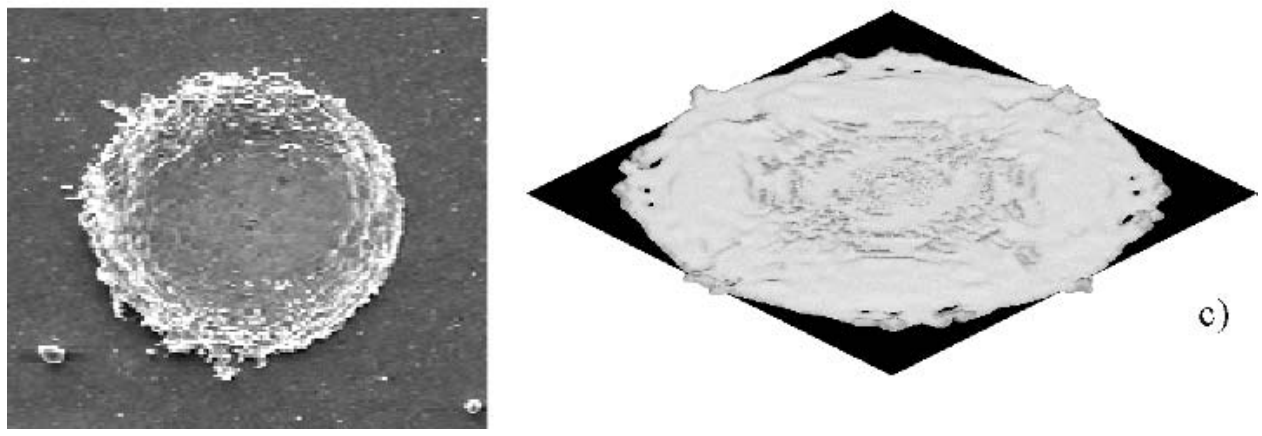

Fig. 7 Nickel splat shapes on a steel plate initially at $400{ }^{\circ} \mathrm{C}$ from (a) experiments, (b) numerical model assuming a contact resistance of $10^{-7} \mathrm{~m}^{2} \mathrm{~K} / \mathrm{W}$, and (c) numerical model assuming a contact resistance of $10^{-6} \mathrm{~m}^{2} \mathrm{~K} / \mathrm{W}$.

steel substrate. Spraying was done with a model SG-100 (Miller Thermal, Appleton, WI) plasma torch operated at a power of $20.5 \mathrm{~kW}$ and an argon flow rate of $1 \mathrm{~g} / \mathrm{s}$. The nickel powder (SF $200 \times 325$, Westaim Corp., Edmonton, Canada) was sieved to a particle size distribution of +53 to $-63 \mu \mathrm{m}$, i.e., particles are bigger than $53 \mu \mathrm{m}$ and smaller than $63 \mu \mathrm{m}$.

A steel shielding plate was placed $100 \mathrm{~mm}$ in front of the plasma gun nozzle, with its center along the same axis as the gun (Fig. 1). A $4 \mathrm{~mm}$ diameter hole was drilled in the center of the plate through which spray particles could pass and reach the test plate that was being coated. Because all of the particles that passed through the hole were near the centerline of the plasma spray, they possessed a relatively narrow temperature and velocity distribution.

In-flight particle conditions were measured at the spraying distance with a commercially available monitoring system, the DPV-2000 (Tecnar Automation, Ltd., Montreal, Canada), which measures the amount of radiation emitted by each hot particle that enters its field of view. It then simultaneously calculates the diameter, velocity, and temperature of the parti- 

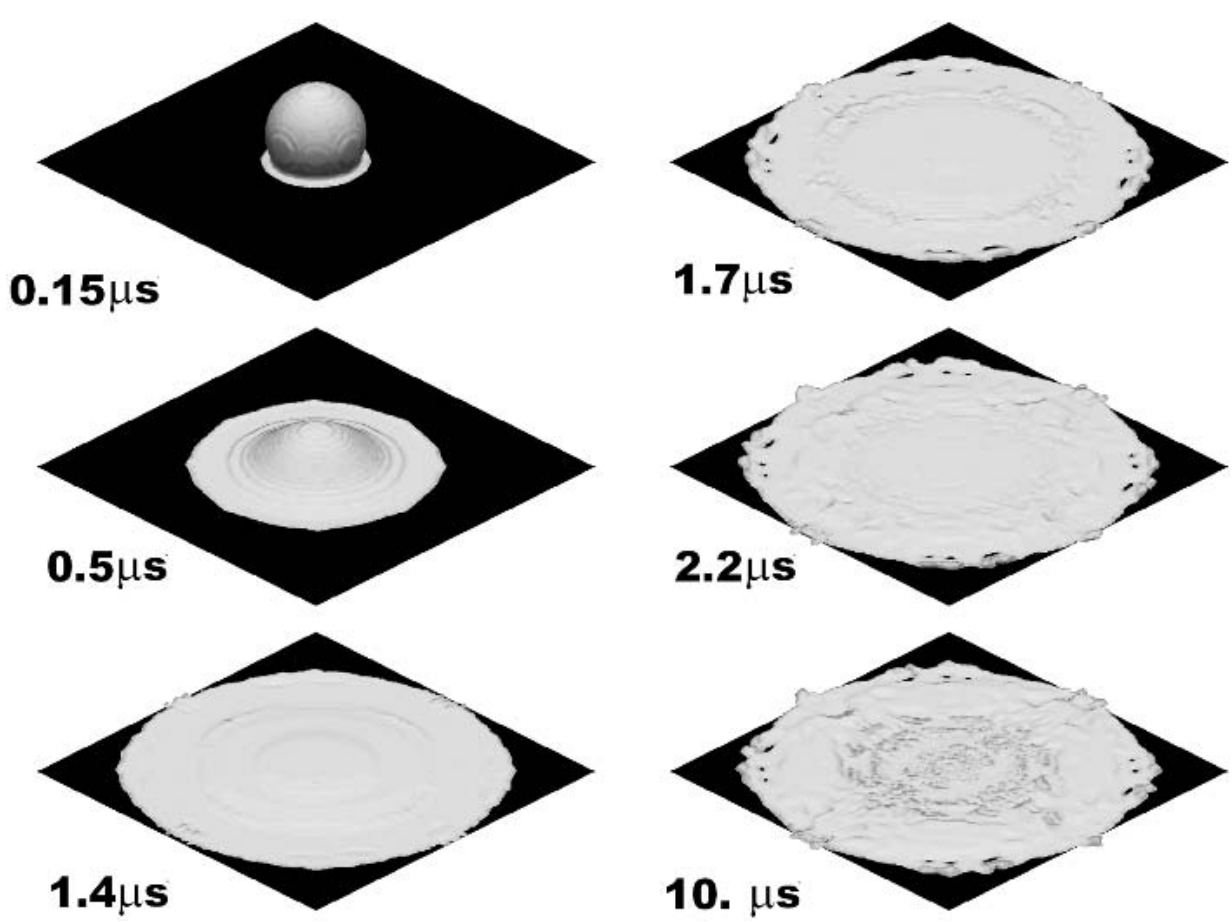

Fig. 8 Simulations showing the impact of a $60 \mu \mathrm{m}$ diameter molten nickel particle at $1600{ }^{\circ} \mathrm{C}$ landing with a velocity of $73 \mathrm{~m} / \mathrm{s}$ on a stainless steel plate initially at a temperature of $400^{\circ} \mathrm{C}$. The contact resistance at the droplet-substrate interface was assumed to be $10^{-6} \mathrm{~m}^{2} \mathrm{~K} / \mathrm{W}$.

cle. In our experiments the particles arrived at the substrate with a mean temperature of $1600 \pm 220^{\circ} \mathrm{C}$ and a velocity of $73 \pm 9$ $\mathrm{m} / \mathrm{s}$.

The nickel particles were sprayed onto a polished stainless steel plate. The average roughness of this substrate was measured with a Surfometer 400 stylus profilometer (Precision Devices Inc., Milan, MI) and found to be $R_{\mathrm{a}}=0.04 \mu \mathrm{m}$. The stainless steel substrate was positioned $50 \mathrm{~mm}$ behind the shielding plate, giving a total spraying distance of $150 \mathrm{~mm}$. The substrate was mounted on a copper block in which was inserted a $500 \mathrm{~W}$ cartridge heater. The temperature of the surface was measured with a J-type thermocouple and regulated by switching on and off power to the heater using a temperature controller. The temperature of the substrate could be varied from 25$640^{\circ} \mathrm{C}$.

The substrates were heated until they achieved the desired temperature; typically it took approximately $10 \mathrm{~min}$ to heat a substrate to $400{ }^{\circ} \mathrm{C}$. This heating was done in air, so the surfaces had time to oxidize. The composition of substrates was tested after they were heated to detect the presence of oxide using an x-ray photoelectron spectrometer (XPS) (MAX-200, Leybold, Cologne, Germany). These results will be discussed below.

A test was started by switching on the plasma gun and introducing the nickel powder into it. Once spray particles were seen passing through the hole in the shielding plate, the heated substrate was rapidly passed through the spray, exposing it to the stream of particles for only a few seconds, which was enough time to collect a few splats. The plate was then examined under a scanning electron and optical microscopes and images of splats were recorded.

\section{Results and Discussion}

\subsection{Effect of Substrate Temperature on Splat Shape}

Figure 2 shows a scanning electron microscope (SEM) micrograph of three nickel splats deposited on a stainless steel surface at a temperature of $290{ }^{\circ} \mathrm{C}$. There is clear evidence of splashing of the droplets. Fingers of solidified materials, which appear to have been created by liquid being thrown out during impact, radiate away from the center. The splats also appear to have ruptured during impact, leaving holes in the middle.

Increasing the substrate temperature significantly reduced splashing. Figure 3 shows nickel splats deposited on a surface at $340{ }^{\circ} \mathrm{C}$. The splats are disk shaped, with only a few fingers projecting out radially. Increasing the temperature even further eliminated most of these fingers. Figure 4 shows splats deposited on a surface at $400{ }^{\circ} \mathrm{C}$, and almost all of them appear to be disk splats, with very little sign of splashing. These results agree well with the previous study of Fukomoto et al., ${ }^{[3]}$ who also observed a sharp transition from splashing to disk splats when nickel particles were sprayed on stainless steel. They measured a transition temperature of approximately $280{ }^{\circ} \mathrm{C}$, somewhat lower than what we observed. However, because they did not report any measurements of particle temperature and velocity, it is difficult to directly compare their data with ours.

\subsection{Splashing Mechanism}

We simulated impact of a molten nickel droplet using our numerical model to determine the mechanism that triggers 

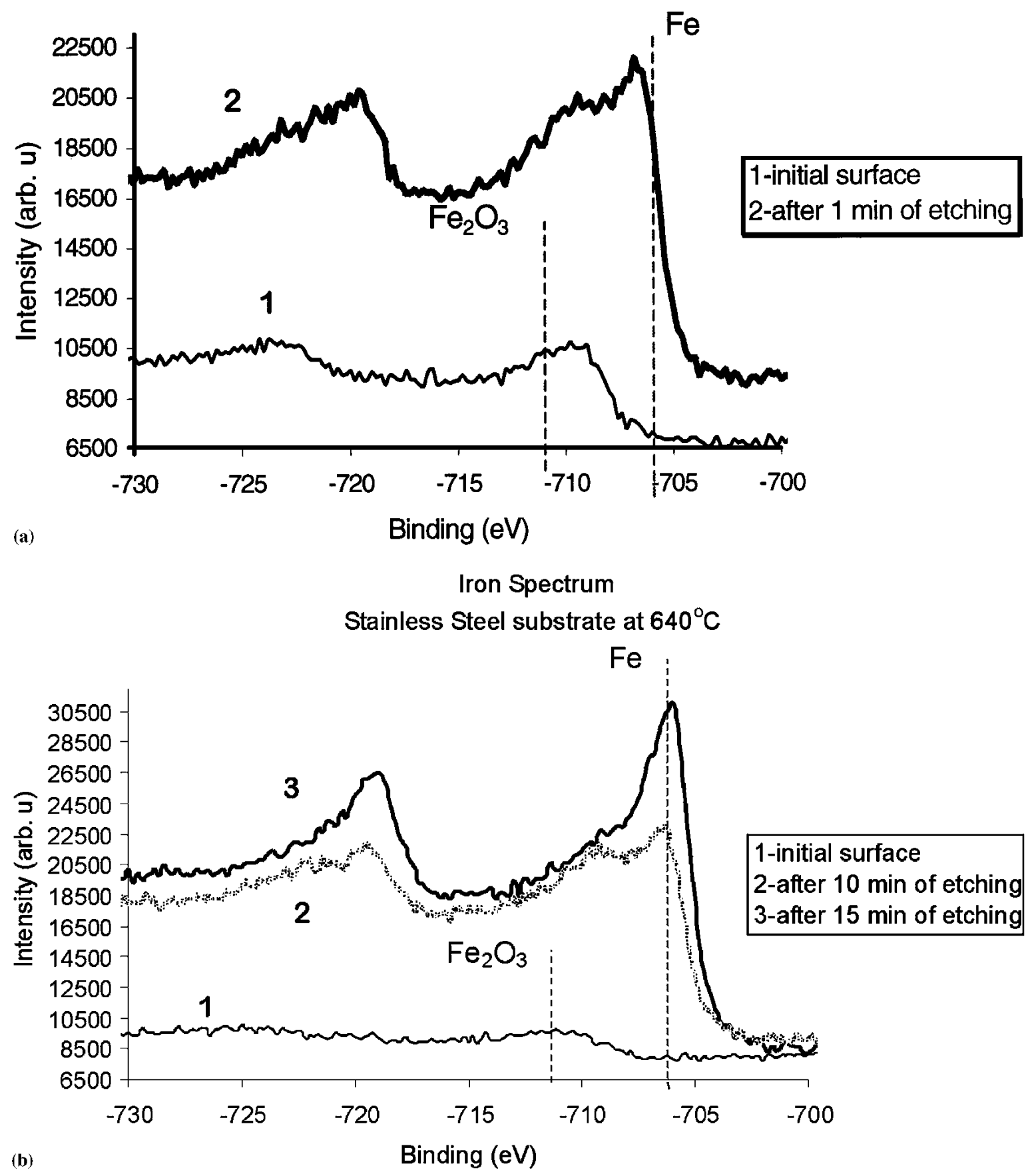

Fig. 9 XPS spectra of stainless steel substrates that were heated to (a) $320^{\circ} \mathrm{C}$ and (b) $640{ }^{\circ} \mathrm{C}$, respectively. The oxide layer on the surface heated to $320^{\circ} \mathrm{C}$ was removed after 1 min of etching with an argon-ion beam, whereas it took 10-15 min of etching to remove the oxide on the surface heated to $640^{\circ} \mathrm{C}$.

splashing. Figure 5 is a sequence of computer-generated images showing successive stages of the impact of a $60 \mu \mathrm{m}$ diameter nickel particle on a stainless steel substrate initially at $290{ }^{\circ} \mathrm{C}$. The droplet was assumed to be at a uniform temperature of 1600 ${ }^{\circ} \mathrm{C}$ prior to impact, which is $150{ }^{\circ} \mathrm{C}$ above the melting point of nickel. The same images are shown in cross section in Fig. 6, with the solid portions of the drop shown in black and the liquid portions shown in white. The time $(t)$ measured from the moment of impact is indicated next to each frame. Calculations were done assuming low thermal contact resistance between the droplet and the substrate, equal to $10^{-7} \mathrm{~m}^{2} \mathrm{~K} / \mathrm{W}$.

Immediately after impact, liquid jetted out from under the 

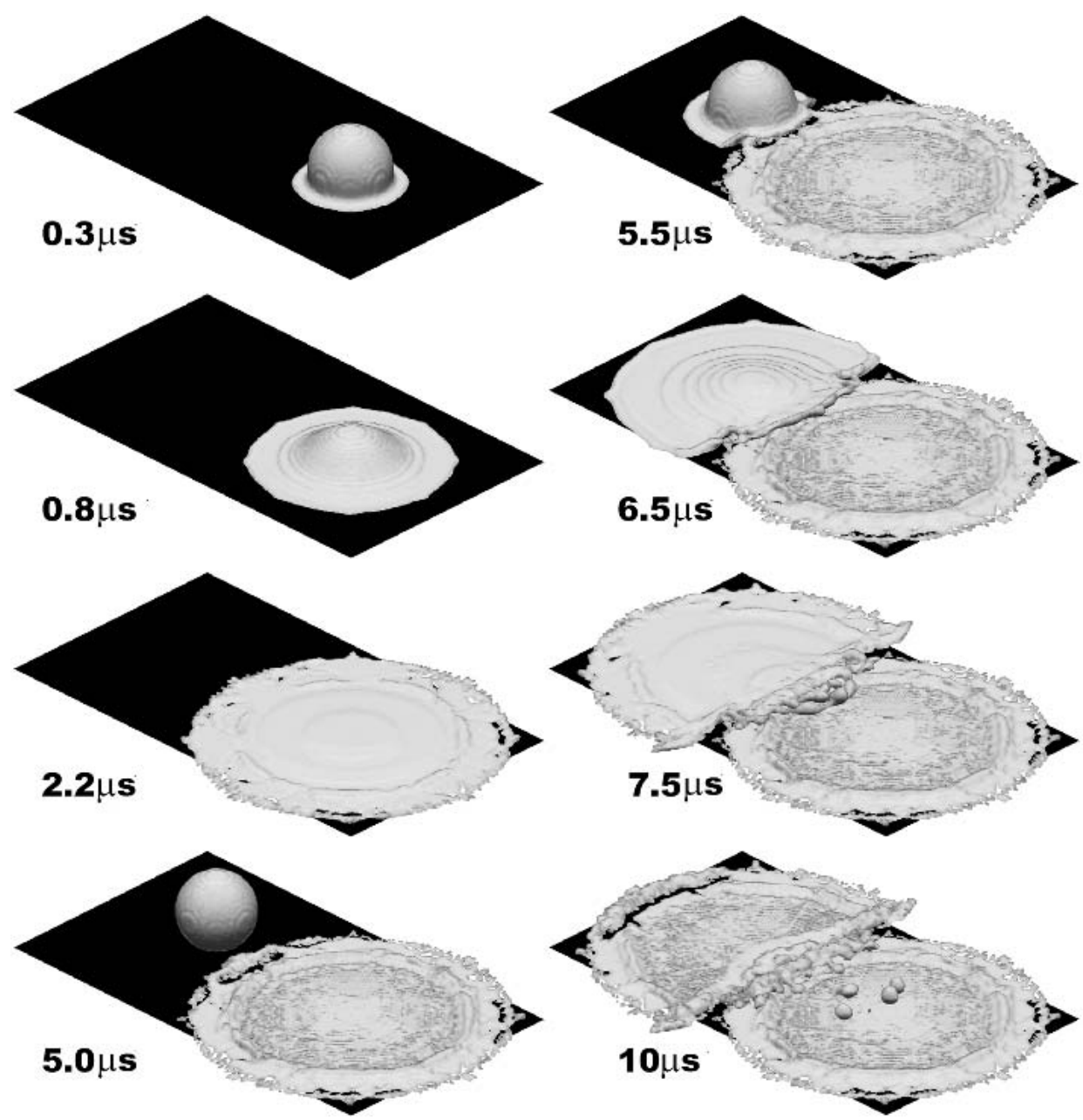

Fig. 10 Simulation images of the impact of two nickel particles $\left(60 \mu \mathrm{m}\right.$ diameter; $48 \mathrm{~m} / \mathrm{s}$ impact velocity; initial temperature $\left.2050{ }^{\circ} \mathrm{C}\right)$ on a stainless steel substrate initially at a temperature of $194{ }^{\circ} \mathrm{C}$. The contact resistance below the droplets was assumed to be $5 \times 10^{-7} \mathrm{~m}^{2} \mathrm{~K} / \mathrm{W}$.

drop and spread in the radial direction, but solidification was fast enough that all the portions of the droplet in contact with the substrate had frozen by $t=0.5 \mu \mathrm{s}$ (Fig. 6). When the bottom layer of liquid solidified, the remaining liquid jetted out over the periphery of the solid splat (Fig. 5 and 6 after $0.8 \mu \mathrm{s}$ ). Because of small variations in the liquid velocity around the periphery of the drop, a fluid instability was created, leading to the formations of fingers of liquid around the drop (Fig. 5, $t=1.4 \mu \mathrm{s}$ ). These fingers grew larger and finally detached to form small satellite droplets. When these droplets touched the substrate they were smeared out as they solidified. The result was formation of solidified fingers around the bulk of the splat as seen 10 $\mu$ s after impact (Fig. 5, $t=10 \mu \mathrm{s}$ ). The final shape of the simulated splat in Fig. 5 resembles the appearance of those we had observed in our experiments when we sprayed a surface at 290 ${ }^{\circ} \mathrm{C}$ (Fig. 2).

\subsection{Effect of Splat-Substrate Thermal Contact Resistance}

We attempted to model the transition to disk splats by increasing the initial substrate temperature to $400{ }^{\circ} \mathrm{C}$ in our simulations. Figure 7(a) shows two images of disk splats that we col- lected after spraying a stainless steel surface initially at $400{ }^{\circ} \mathrm{C}$. Figure 7(b) shows the final shape of our simulated splat, calculated assuming an initial surface temperature of $400{ }^{\circ} \mathrm{C}$ and a thermal contact resistance $R_{\mathrm{c}}=10^{-7} \mathrm{~m}^{2} \mathrm{~K} / \mathrm{W}$. The droplet showed less splashing than it did in the previous simulation of impact on a surface at $290{ }^{\circ} \mathrm{C}$ (Fig. 5), but there were still a significant number of fingers around it. The reason splashing diminishes on a hotter surface is that solidification is delayed and therefore the fluid flow is not disturbed as much by a frozen layer.

Splashing could be eliminated completely in our simulations if the droplet did not start solidifying until it had finished spreading. The onset of solidification could be delayed if we increased the value of the thermal contact resistance between the drop and the substrate, thereby reducing heat transfer. Figure $7(\mathrm{c})$ shows the final splat shape on a surface at a temperature of $400{ }^{\circ} \mathrm{C}$, assuming a thermal contact resistance $R_{\mathrm{c}}=10^{-6} \mathrm{~m}^{2} \mathrm{~K} / \mathrm{W}$, which was an order of magnitude larger than that used previously. The splat was disk shaped, with no splashing, looking much like those observed experimentally (Fig. 7a).

Extensive trials with the numerical model confirmed that solidification is necessary to trigger splashing in a nickel droplet of the size found in thermal spray applications ( $<100 \mu \mathrm{m}$ diameter). 


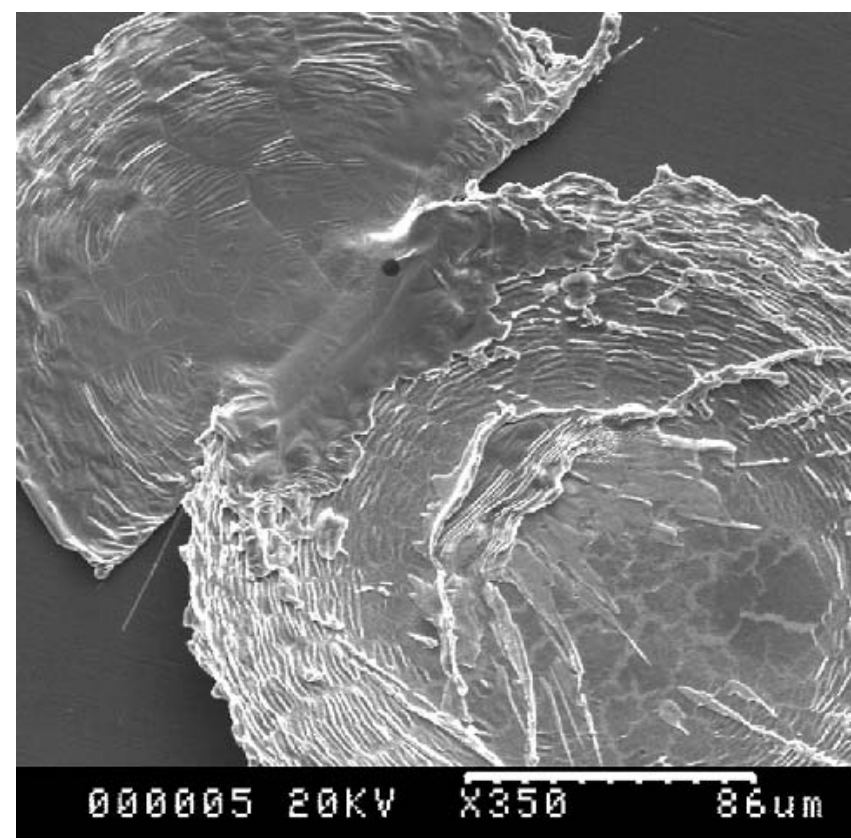

Fig. 11 Micrograph of two nickel particles deposited on a stainless steel surface at $400{ }^{\circ} \mathrm{C}$

Figure 8 shows the impact of a $60 \mu \mathrm{m}$ diameter nickel droplet landing on a stainless steel substrate at a temperature of $400{ }^{\circ} \mathrm{C}$ with thermal contact resistance $R_{\mathrm{c}}=10^{-6} \mathrm{~m}^{2} \mathrm{~K} / \mathrm{W}$. Heat transfer from the droplet was so low that it spread without solidifying and formed a disk splat. Solidification was delayed until droplet spreading was almost complete. We tried to induce splashing in the simulated droplet by artificially perturbing the velocity field, but were unable to do so; surface tension forces were so strong that they damped out the perturbations and prevented the growth of fingers in the spreading liquid. Changing the liquid-solid contact angle also had little effect on splashing, because inertial forces drive liquid flow after impact and capillary forces have little effect.

Our simulations showed that splashing could not be suppressed by increasing the substrate temperature alone, but also required an increase in contact resistance. We conjectured that heating the substrate creates an oxide layer on the surface that increases the contact resistance. To confirm this hypothesis we analyzed the oxide scale on the test surfaces using an XPS in combination with Ar-ion sputtering of the substrate. The spectrometer monitors surface composition (in particular, iron, chromium, and oxygen), whereas the ion etching gradually removes oxide from the steel surface. The time taken to etch away the oxide layer and expose a bare iron surface is therefore an indication of the thickness of the oxide layer.

We measured the oxide layers on stainless steel surfaces that were heated in air and then allowed to cool. Figure 9 shows typical XPS spectra for two substrates, one heated to a maximum temperature of $320^{\circ} \mathrm{C}$ and the other heated to $640^{\circ} \mathrm{C}$. The initial scan of the surface preheated to $320^{\circ} \mathrm{C}$ (Fig. 9a) shows the presence of $\mathrm{Fe}_{2} \mathrm{O}_{3}$ (which has a binding energy of $711 \mathrm{eV}$ ) on the surface. It took approximately 1-1.5 min of etching to remove this oxide layer and detect the presence of $\mathrm{Fe}$, which is shown by a peak on the spectrum at $707 \mathrm{eV} .^{[17]}$ The surface heated to 640 ${ }^{\circ} \mathrm{C}$ had a much thicker oxide layer: it took on average of 10-15
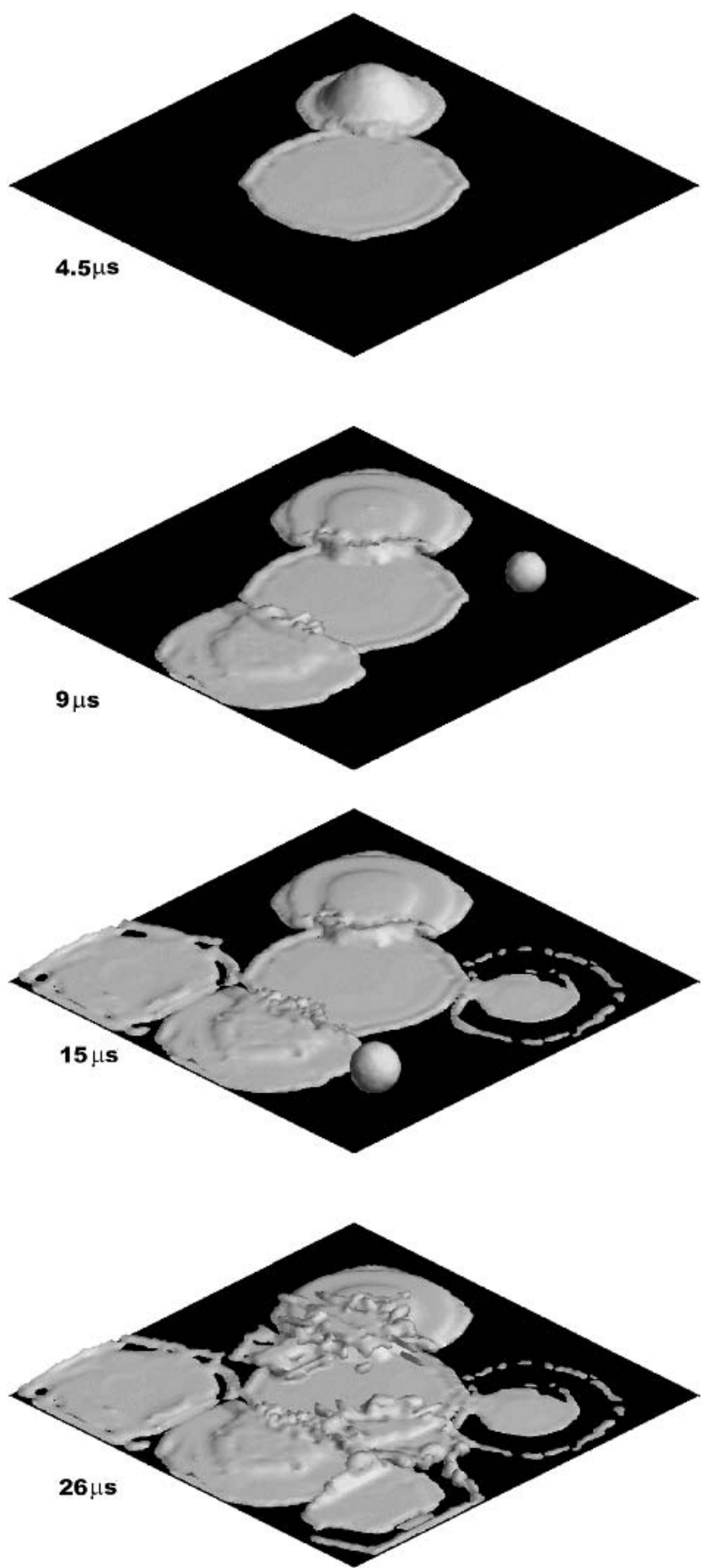

Fig. 12 Simulation of the impact of nine consecutive nickel particles on a $500 \times 500 \mu \mathrm{m}$ steel plate initially at $20^{\circ} \mathrm{C}$. Particle diameters range from $40-80 \mu \mathrm{m}$ and their impact velocities from $40-80 \mathrm{~m} / \mathrm{s}$. The particles were at temperatures from $1600-2000{ }^{\circ} \mathrm{C}$, well above their melting point. Contact resistance between the particles and substrate was assumed to be $10^{-7} \mathrm{~m}^{2} \mathrm{~K} / \mathrm{W}$.

min of etching to remove the oxide and reach bare metal. Therefore, preheating the surface to $640{ }^{\circ} \mathrm{C}$ creates an oxide layer an order of magnitude greater than that on a surface at $320^{\circ} \mathrm{C}$. We would expect a corresponding increase in thermal contact resistance. 


\subsection{Splat Shape from Multiple Impact}

Solidification inside a spreading droplet can trigger splashing. However, other protrusions on the surface can also make a droplet splash, such as scratches on the surface. ${ }^{[3]}$ The presence of an already solid splat under an impacting droplet can also create an instability that causes droplet splashing. Figure 10 shows simulations of the sequential impact of two nickel droplets, both $60 \mu \mathrm{m}$ in diameter and with impact velocities of $48 \mathrm{~m} / \mathrm{s}$, landing on a stainless steel surface at $194{ }^{\circ} \mathrm{C}$. The second droplet landed $5 \mu$ s after the first, with its center offset by $140 \mu \mathrm{m}$ from that of the first droplet. Contact resistance under both drops was assumed to be $5 \times 10^{-7} \mathrm{~m}^{2} \mathrm{~K} / \mathrm{W}$. The first droplet landed and spread without any significant splashing, forming a disk splat (Fig. $10, t=2.2 \mu \mathrm{s}$ ). The second droplet was introduced after the first was completely solidified, and it landed near the edge of the first splat. The spreading sheet of liquid hit the solidified splat and was in part directed sideways, as seen in Fig. 10 after $t=5.5 \mu \mathrm{s}$. The remainder of the liquid sheet jetted upward over the solidified splat (Fig 10, $t=6.5 \mu \mathrm{s}$ ) and began to fragment (Fig. 10,t=7.5 $\mu \mathrm{s}$ ). The effect of liquid breakup could be seen as small droplets flying on top of the splats (Fig. 10, $t=10 \mu \mathrm{s}$ ). We saw evidence of this type of behavior in our experiments: Fig. 11 shows two splats deposited next to each other on a surface at $400{ }^{\circ} \mathrm{C}$. The first splat is disklike, but the second splashed after hitting the edge of the first. Streaks of splashed material are visible on the surface of the first splat.

Predicting the shapes of splats in a thermal spray coating is a complex problem, because their final shape is determined by their interactions with each other and with the solid substrate. We simulated coating deposition using our numerical model. Figure 12 shows the result of a simulation in which nine nickel particles were deposited sequentially in random locations on a $500 \times 500 \mu \mathrm{m}$ stainless steel substrate initially at $20^{\circ} \mathrm{C}$. Particle diameter ranged from 40 to $80 \mu \mathrm{m}$ and impact velocities ranged from 40 to $80 \mathrm{~m} / \mathrm{s}$. Particles were initially superheated, with temperatures varying from 1600 to $2000{ }^{\circ} \mathrm{C}$, and landed at time intervals of $3 \mu \mathrm{s}$. Contact resistance was assumed to be $10^{-7} \mathrm{~m}^{2} \mathrm{~K} /$ W. Four successive stages during deposition are shown in Fig. 12. A variety of splat shapes can be seen in the simulated images, including disk splats, splashing where splats contacted each other, and the formation of annular rings around splats that broke up during impact.

\section{Conclusions}

Nickel particles sprayed onto a stainless steel plate splash if the substrate temperature is below $300^{\circ} \mathrm{C}$, but form round disk splats on substrates heated above $400{ }^{\circ} \mathrm{C}$. Simulations showed that solidification in the spreading droplet triggers the formation of fingers: a droplet that spreads completely before it starts to freeze will not splash. Increasing the substrate temperature to $400{ }^{\circ} \mathrm{C}$ in our simulations did not sufficiently reduce heat transfer from the droplet to the substrate to eliminate splashing. However, increasing the thermal contact resistance by an order of magnitude delayed the onset of splashing and gave disk splats. We measured the thickness of the oxide layer on the test surfaces used in our experiments and confirmed that heating them creates an oxide layer on the surface that increases the thermal contact resistance.

Splashing of droplets can also be triggered by other protrusions on the test surface, such as previously deposited splats. We modeled the interaction of two or more droplets deposited sequentially on a surface and observed a variety of splat shapes formed as droplets spread and solidified.

\section{References}

1. M. Vardelle, A. Vardelle, A.C. Leger, P. Fauchais, and D. Gobin: "Influence of Particle Parameters at Impact on Splat Formation and Solidification in Plasma Spraying Process," J. Thermal Spray Technol., 1995, 4, pp. 50-58.

2. L. Bianchi, F. Blein, P. Lucchese, M. Vardelle, A. Vardelle, and P. Fuchais: "Effect of Particle Velocity and Substrate Temperature on Alumina and Zirconia Splat Formation" in Thermal Spray Industrial Applications, C.C. Berndt and S. Sampath, ed., ASM International, Materials Park, OH, 1994, pp. 569-74.

3. M. Fukomoto, Y. Huang, and M. Ohwatari: "Flattening Mechanism in Thermal Sprayed Particle Impinging on Flat Substrate" in Thermal Spray: Meeting the Challenges of the 21st Century, C. Coddet, ed., ASM International, Materials Park, OH, 1998, pp. 401-6.

4. C.J. Li, J.L. Li, W.B. Wang, A. Ohmori, and K. Tani: "Effect of Particle Substrate Materials Combinations on Morphology of Plasma Sprayed Splats" in Thermal Spray: Meeting the Challenges of the 21st Century, C. Coddet, ed., ASM International, Materials Park, OH, 1998, pp. 48187.

5. C. J. Li, J.L. Li, and W.B. Wang: "The Effect of Substrate Preheating and Surface Organic Covering on Splat Formation" in Thermal Spray: Meeting the Challenges of the 21st Century, C. Coddet, ed., ASM International, Materials Park, OH, 1998, pp. 473-80.

6. J. Pech, B. Hannoyer, A. Denoirjean, and P. Fauchais: "Influence of Substrate Preheating Monitoring on Alumina Splat Formation in DC Plasma Process" in Thermal Spray: Surface Engineering via Applied Research, C.C. Berndt, ed., ASM International, Materials Park, OH, 2000, pp. 759-65.

7. N. Sakakibara, H. Tsukuda, and A. Notomi: "The Splat Morphology of Plasma Sprayed Particle and the Relation to Coating Property" in Thermal Spray: Surface Engineering via Applied Research, C.C. Berndt, ed., ASM International, Materials Park, OH, 2000, pp. 481-87 and 75358.

8. M. Pasandideh-Fard and J. Mostaghimi: "On the Spreading and Solidification of Molten Particles in a Plasma Spray Process: Effect of Thermal Contact Resistance," Plasma Chem. Plasma Process., 1996, 16, pp. 83S-98S.

9. Z. Zhao, D. Poulikakos, and J. Fukai: "Heat Transfer and Fluid Dynamics During the Collision of a Liquid Droplet on a Substrate-I. Modeling," Int. J. Heat Mass Transfer, 1996, 39, pp. 2771-89.

10. M. Bertagnolli, M. Marchese, G. Jacucci, I. St. Doltsinis, and S. Noelting: "Thermomechanical Simulation of the Splashing of Ceramic Droplets on a Rigid Substrate," J. Comput. Phys., 1997, 133, p. 205 .

11. J.M. Waldvogel and D. Poulikakos: "Solidification Phenomena in Picoliter Size Solder Droplet Deposition on a Composite Substrate," Int. J. Heat. Mass Transfer, 1997, 40, pp. 295-309.

12. M. Pasandideh-Fard, R. Bhola, S. Chandra, and J. Mostaghimi: "Deposition of Tin Droplets on a Steel Plate: Simulations and Experiments," Int. J. Heat Mass Transfer, 1998, 41, pp. 2929-45.

13. M. Bussmann, J. Mostaghimi, and S. Chandra: "On a ThreeDimensional Volume Tracking Model of Droplet Impact," Phys. Fluids, 1999, 11, 1406-17.

14. Y. Cao, A. Faghri, and W.S. Chang, "A Numerical Analysis of Stefan Problems for Generalized Multi-Dimensional Phase-Change Structures 
Using the Enthalpy Transforming Model," Int. J. Heat Mass Transfer, 1989, 32, pp. 1289-98.

15. M. Pasandideh-Fard: "Droplet Impact and Solidification in a Thermal Spray Process," Ph.D. Thesis, University of Toronto, 1998.

16. V. Voller and M. Cross: "An Explicit Numerical Method to Track a
Moving Phase Change Front," Int. J. Heat Mass Transfer, 1983, 26, pp. $147-50$.

17. J.F. Moulder, W.F. Stickle, P.E. Sobol, and K.D. Bomben, Handbook of $X$-ray Photoelectron Spectroscopy, Perkin-Elmer Corp., Physical Electronics Division, Eden Prairie, MN, 1992.

.

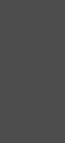

